\title{
Relay Selection Methods for Maximizing the Lifetime of Wireless Sensor Networks
}

\author{
Fábio Engel ${ }^{1}$, Taufik Abrão ${ }^{1} \&$ Lajos Hanzo ${ }^{2}$ \\ ${ }^{1}$ Electrical Engineering and Computer Departments, State University of Londrina, PR, Brazil. fabioengel@gmail.com; taufik@uel.br \\ ${ }^{2}$ School of Electronics and Computer Science, University of Southampton, SO17 1BJ Southampton, U.K. Iheecs.soton.ac.uk.
}

\begin{abstract}
Combined analytical and fuzzy techniques are proposed for improving the battery lifetime, performance, as well as energy efficiency of wireless sensor networks (WSNs) with the aid of efficient relay selection methods. We determine the best relay selection method by striking an appealing performance versus network lifetime tradeoff. Furthermore, the beneficial regions of cooperation are determined considering asymmetric traffic scenarios, where relaying provides energy saving.
\end{abstract}

\section{Introduction AND System Model}

Wireless sensor networks (WSNs) consist of multiple autonomous devices, which are capable of sensing, processing and communication. The applications are numerous, for example, in health care, where the patient's bodily functions can be monitored by miniature devices and then reported to a central processing unit [1]. In the military domain the self organization capability of sensor networks is beneficial, since the sensors may have to operate in a harsh environment, under constant changes in their topology. Sensors can also be used in commercial applications, such as monitoring of industrial production steps and parameters, such as pressure, temperature and flow [2].

Sensor nodes often operate in regions with difficult or no access for humans, for example in contaminated areas. It is expected then that the sensor nodes have a long operating period without their energy reserve being exhausted. Hence the efficient use of energy is paramount. The total power consumption is dominated by three fundamental units that form the sensor node, namely: a sensing unit, a processing unit and a transmission unit.

While for sensing the power used varies according to the nature of the application, typically the power consumption of the transmission unit is significantly higher than that of the processing unit. As noted in [2], assuming a Rayleigh fading channel, i.e. a channel with no line of sight (NLOS), and a path-loss with an exponent of $\xi=4$, the energy dissipated by transmitting $1 \mathrm{~KB}$ of data to a distance of 100 meters is approximately the same as that required for executing 3 million instructions by a processor that performs 100 million instructions per second.

In this work we consider a system model based on Fig. 1 , which consists of a source node ( $\mathrm{SN})$, denoted by $s$, a destination node (DN) $d$ and $K$ relay nodes (RNs) denoted by $R_{k}, 1 \leq k \leq K$, which are randomly distributed between $s$ and $d$. The power gain of the wireless channel, namely $\left|h_{i, j}\right|^{2}$, where $i, j$ represent the index of the source, relay or destination nodes, incorporates the effects of large-scale fading, related to the propagation characteristics of the signal over long distances, and the Rayleigh-distributed small-scale fading. Hence, the power gain of the SR, RD and SD links are respectively:

$$
\begin{gathered}
\left|h_{s, i}\right|^{2}=\kappa \cdot d_{s, i}^{-2 \xi} \cdot v_{s, i} ; \quad\left|h_{i, d}\right|^{2}=\kappa \cdot d_{i, d}^{-2 \xi} \cdot v_{i, d} \\
\left|h_{s, d}\right|^{2}=\kappa \cdot d_{s, d}^{-2 \xi} \cdot v_{s, d}
\end{gathered}
$$

with $\kappa$ being a constant defined by the antenna gain, carrier frequency and other system parameters; $\xi$ is the path loss component; $v$ is an exponentially distributed random variable; $d_{s, i}, d_{i, d}$ and $d_{s, d}$ are the respective distances.

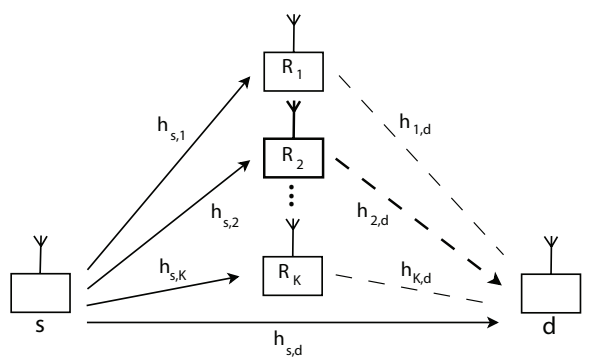

Fig. 1. Half-duplex dual-hop relaying topology. Solid arrows: broadcast transmission in slot-1; select (bold) dashed arrow: transmission in slot-2.

With the goal of quantifying the energy efficiency (EE) of diverse relay selection schemes, computer simulations were carried out, which are described in Section III. The choice of the suitable relay obeys the general steps of: a) SN transmits a Request-to-Send (RTS1) packet in its broadcast mode, so that each relay can estimate the channel gain $h_{s, i}$; b) RNs send a RTS2 packet to the DN containing an indication of the channel quality. Thus, DN can now estimate the channel gain $h_{i, d} ; \mathrm{c}$ ) A relay selection algorithm is applied, and DN transmits in its broadcast mode the index of the selected relay; d) SN and DN establish communication via the RN selected in the previous step.

Energy \& Spectral Efficiency in Relay Channels. Within the scope of energy efficiency (EE), a widely used metric is the bit-energy: $E_{\mathrm{B}}=(\Sigma P) / R\left(1-P_{\mathrm{e}}\right)=\varepsilon / n_{b}\left(1-P_{\mathrm{e}}\right)$, in $\left[\mathrm{J} /\right.$ bits], where $P_{\mathrm{e}}$ is the bit-error probability, $\Sigma P$ is the total power required to transmit the information to the $\mathrm{DN}$, including the transmit power and the power consumption dissipated by the processing circuitry; $R$ is the information bit rate [bits/sec]; the total energy dissipated by the transmission is given by $\varepsilon$ in [Joules] and $n_{b}$ is the number of information bits transmitted in one second.

On the other hand, the spectral efficiency (SE) of a system quantifies how efficiently the spectrum is explored, which is given by the ratio of the information rate reliably detected over a given bandwidth $B: \mathcal{S}=R\left(1-P_{\mathrm{e}}\right) / B$, in $[\mathrm{bits} / \mathrm{s} / \mathrm{Hz}$ ].

In a cooperative system, the spectral efficiency $\mathcal{S}^{\text {coo }}$ is an important parameter to be evaluated and optimized. As a reference, let us consider the $\mathrm{SE} \mathcal{S}$ of direct communication 
between the SN and DN. Bearing in mind that we have to create two time-slots (TS), namely one for broadcasting and one for cooperation, the spectral efficiency of each hop should not be lower than $2 \cdot \mathcal{S}$, so that the cooperative system achieves an end-to-end spectral efficiency of $\mathcal{S}$; Explicitly, both the SR and RD links should satisfy:

$$
\mathcal{S}_{s, r}^{\mathrm{coo}} \geq 2 \cdot \mathcal{S} \quad \text { and } \quad \mathcal{S}_{r, d}^{\mathrm{coo}} \geq 2 \cdot \mathcal{S} \quad[\mathrm{bits} / \mathrm{s} / \mathrm{Hz}] .
$$

For simplicity, the overall SE of both the uplink and downlink of the cooperative system are assumed to be: $\mathcal{S}\left[\frac{\mathrm{bits}}{\mathrm{s} \cdot \mathrm{Hz}}\right]$.

Channel Power Gain Constraints and Normalization. Let us assume that a large number of RNs are randomly distributed. According to the position of each RN the channel gain $h_{i, d}$ can be estimated. For the sake of ensuring that the normalized channel gains are independent of the cell topology, a normalization factor given by $k d_{i, \max }^{-\xi}$ has also been applied, where $d_{i, \max }$ is the maximum distance between the $i$ th $\mathrm{RN}$ and the DN. Thus, the normalized channel power gain becomes:

$$
\left|h_{n}\right|^{2}=d_{i, d}^{-2 \xi} \cdot d_{i, \max }^{2 \xi} \cdot v_{i, d} .
$$

WSNs Asymmetric Traffic. We rely on an asymmetric traffic model capable of representing an unbalanced traffic between the DownLink (DL) and UpLink (UL) directions. In general, the WSN UL traffic is higher; hence, we define the trafficasymmetry factor as:

$$
\zeta=L_{\mathrm{UL}} / L_{\text {total }}=L_{\mathrm{UL}}\left(L_{\mathrm{UL}}+L_{\mathrm{DL}}\right)^{-1},
$$

where $L_{\text {total }}$ is the total traffic load given by the sum of the traffic $L_{\mathrm{UL}}$ generated in the UL direction and DL direction $L_{\mathrm{DL}}$. Note that although the traffic in the UL direction of a WSN is typically higher, since the RNs transmit the information acquired, the reverse situation might also occur, for instance when updated parameters are sent to the SN.

The power consumption of the $i$ th WSN transmitter is given by the sum of the transmission signal power $P_{i}$ (dynamic term dependent on the level of relay activities) and the fixed power dissipated by the circuitry of the SNs and RNs:

$$
\mathcal{P}=\sum_{i=1}^{K+1} P_{i}+P_{C}=P_{T}+P_{C} \leq P_{\max } \quad[W],
$$

where we have $P_{C}=(K+1) P_{\mathrm{C}, \mathrm{n}}$ associated with a fixed power circuitry dissipation per node $P_{\mathrm{C}, \mathrm{n}}$, while $P_{\max }$ is the maximum battery power available at the SN and RNs.

Since in WSNs the DN must store all the information obtained from the other nodes, it is expected that it consumes more energy. Hence the DN imposes different challenges and has different specifications from those of the SN and RN. Thus, herein the cost of energy dissipated by the DN is ignored.

\section{A. Energy Consumption Model in Relay Channels}

Based on (4) and (5), the total energy consumed by the SN and a single $\mathrm{RN}$ is $\varepsilon^{\mathrm{coo}}=P_{\mathrm{S}}^{\mathrm{U}} T_{\mathrm{S}}^{\mathrm{U}}+P_{\mathrm{R}}^{\mathrm{U}} T_{\mathrm{R}}^{\mathrm{U}}+P_{\mathrm{R}}^{\mathrm{D}} T_{\mathrm{R}}^{\mathrm{D}}[\mathrm{J}]$, while the associated energy-per-bit becomes:

$$
E_{\mathrm{B}}^{\mathrm{coo}}=\left[\zeta\left(P_{\mathrm{S}}^{\mathrm{U}}+P_{\mathrm{R}}^{\mathrm{U}}\right)+(1-\zeta) P_{\mathrm{R}}^{\mathrm{D}}\right] / 2 \mathcal{S} B \quad[\mathrm{~J} / \mathrm{bit}],
$$

where $P_{\mathrm{S}}^{\mathrm{U}}$ and $P_{\mathrm{R}}^{\mathrm{U}}$ is the power of the $\mathrm{SN}$ and $\mathrm{RN}$ used for UL data transmission, respectively; $P_{\mathrm{R}}^{\mathrm{D}}$ is the power of the
$\mathrm{RN}$ in the DL; $T_{\mathrm{S}}^{\mathrm{U}}, T_{\mathrm{R}}^{\mathrm{U}}$ and $T_{\mathrm{R}}^{\mathrm{D}}$ are the respective transmission durations; $\mathcal{S}=\frac{R\left(1-P_{\mathrm{e}}\right)}{B} \approx \frac{L_{\text {total }}}{T_{\text {total }} \cdot B}$ is the spectral efficiency of the system expressed in $\left[\frac{\mathrm{bits}}{\mathrm{s} \cdot \mathrm{Hz}}\right]$, which is assumed to obey $P_{\mathrm{e}}<<1$; the network bandwidth is $B$ in $[\mathrm{Hz}]$, while the UL and DL transmit periods $T^{\mathrm{U}}$ and $T^{\mathrm{D}}$, respectively, are defined by the relations: $T^{\mathrm{U}}=\frac{L_{\mathrm{UL}}}{2 \mathcal{S} B}$ and $T^{\mathrm{D}}=\frac{L_{\mathrm{D}}}{2 \mathcal{S} B}$, respectively, with $T_{\text {total }}=T^{\mathrm{U}}+T^{\mathrm{D}}$.

The bit error rate (BER) for $M-\mathrm{QAM}$ associated with Gray mapping can be approximated by [3]:

$$
P_{\mathrm{e}}(\gamma) \approx 0.2 \exp [-1.5 \gamma /(M-1)],
$$

where the signal-to-noise ratio (SNR) at the receiver is defined as:

$$
\gamma_{i}=P_{i}\left|h_{i, j}\right|^{2} / N_{0} B
$$

Defining the Quality of Service (QoS) in terms of the maximum tolerable BER, we obtain the transmit power required for achieving this QoS, where substituting (8) into (7) yields:

$$
P_{i}=2 \ln \left(5 P_{e}\right) N_{0} B(1-M) / 3\left|h_{i, j}\right|^{2} .
$$

However, the ratio of the energy per information bit and the noise spectral density $\left(E_{b} / N_{0}\right)$ can be reduced by the coding gain $\mathcal{G}$ of the error correction code (ECC). Thus, the transmission power required by $M-\mathrm{QAM}$ combined with trellis coding can be expressed as [4]:

$$
P_{i_{\mathrm{C}}}=\varrho P_{i} / \mathcal{G}=2 \varrho \ln \left(5 P_{e}\right) N_{0} B\left(1-2^{\mathcal{S} / \varrho}\right) / 3\left|h_{i, j}\right|^{2} \mathcal{G},
$$

where $\varrho<1$ is the code rate of the trellis encoder. According to [4], the BER of cooperative communication using the Decode-and-Forward (DF) protocol is given by [5]:

$$
P_{e}=1-\left[1-P_{e}\left(\gamma_{s}\right)\right]\left[1-P_{e}\left(\gamma_{d}+\gamma_{s d}\right)\right],
$$

where $\gamma_{s}, \gamma_{d}$ and $\gamma_{s d}$ represent the SNR of the SR, RD and SD links, respectively. The optimal power allocation for the DF protocol is obtained as a function of SNR [6], satisfying the following relationship: $\gamma_{s}=\gamma_{d}+\gamma_{s d}$. Therefore, the BER of the SR and RD links shall be the same, i.e., $P_{e}\left(\gamma_{s}\right)=$ $P_{e}\left(\gamma_{d}+\gamma_{s d}\right)=1-\sqrt{1-P_{e}}$.

\section{B. Relay Location: Cooperative Regions}

In cooperative communication one of the most important factors determining the achievable gain is the location of the nodes, especially that of the RN. Aiming for determining the areas, where the adoption of a $\mathrm{RN}$ is considered advantageous, a common approach relies on the channel power gains, ignoring the short-term fading, and then obtaining the cooperative energy-gain defined as the percentage of energy saved with the aid of cooperation, while aiming for the same spectral efficiency as the direct transmission between the SN and DN [4], yielding:

$$
E_{\text {save } \%}=100 \cdot\left(E^{\mathrm{dir}}-E^{\mathrm{coo}}\right) / E^{\mathrm{dir}} \quad[\%],
$$

with $E^{\text {dir }}$ and $E^{\text {coo }}$ being the energy dissipation of the direct and cooperative mode, respectively, which are detailed in Section II-B. Fig. 2 illustrates the contour lines that delimit the cooperative energy-gain regions $\left(E_{\mathrm{save} \%}>0\right)$, when considering the traffic-asymmetry factors of $\zeta \in\{0.1 ; 0.3 ; 0.5 ; 0.7 ; 0.9\}$. Note that the ellipsoid area which determines the cooperation 
region increases for $\zeta \rightarrow 1$. For instance, considering an asymmetric traffic in the sink-relay-source direction $(\zeta=0.1)$, the inner-most ellipsoid area indicates the most beneficial RN location (in terms of maximum cooperative energy-gain) in the vicinity of the point "RS". As expected for $\zeta=0.5$, the best "RS" position is almost equidistant from the source and sink nodes. Activating relays positioned inside the small area centered around the "RS" point facilitates energy-gains higher than $50 \%$ in the cooperative mode. On the other hand, in the presence of relays located outside the ellipsoids of Fig. 2 implies that the cooperative mode is not useful in terms of energy saving, when compared to the direct transmission mode dispensing with relaying.

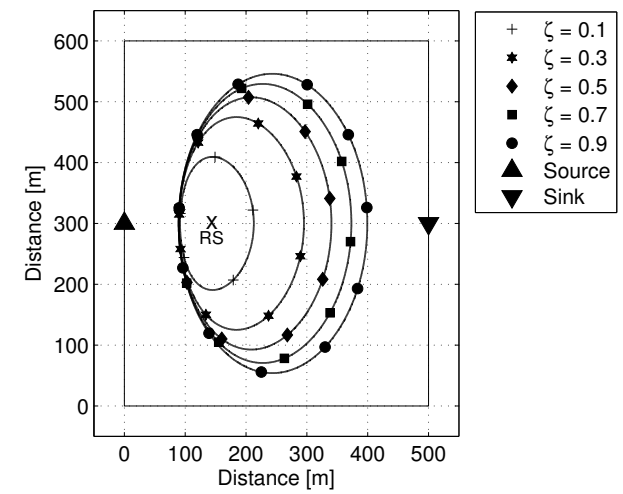

Fig. 2. Cooperative energy-gain $E_{\text {save }_{\%}}>0$ limit regions for different asymmetric traffic $\zeta$ values.

\section{Classical Relay Selection Approaches}

The relays used in cooperative sensor networks aim for improving both the network's battery lifetime $\mathcal{L}$ and information rate. The network's battery lifetime can be quantified by the time duration in which the first node belonging to the network starts failing, because its energy reserve has been depleted.

Relay-selection algorithms differ in their objective function (OF), since the multi-component OFs may incorporate conflicting factors, which cannot always be combined; for instance, when our goal is the reduction of the energy consumption, the RNs having the best channel conditions should be chosen, resulting in the depletion of their energy reserve; consequently, the network's lifetime will be negatively affected.

A Random Relay Selection $(R-R S)$ algorithm randomly selects the $\mathrm{RN}$ to be used for relaying the signal. When receiving a Negative Acknowledgement (NACK) message each time interval $t$, the $t$-th relay acts as an auxiliary SN. As an advantage, this technique aims for equitably distributing the power dissipated by relaying, and thus the lifetime of the batteries of all network nodes also decays equally and slower, hence avoiding the early collapse of the entire network owing to a single device without a charged battery [7]. The disadvantage is however that by activating a RN without considering any additional information, the RN might be far from the DN or, might experience hostile channel conditions, or both.

The Maximum Harmonic Mean Relay Selection (MHM-RS) method [8] takes into account the channel quality between the SR and RD node for deciding, which particular relay will be selected for transmission. The RN associated with the highest $h_{s, i}$ and $h_{i, d}$ gain between the $\mathrm{SN}$ and DN is selected, which is formulated as:

$$
r=\arg \max _{i \in \mathcal{K}} H\left(\left|h_{s, i}\right|^{2},\left|h_{i, d}\right|^{2}\right),
$$

where $\mathcal{K}$ is the $\mathrm{RN}$ set, and the function $H(\cdot)$ is given by the harmonic mean of $a$ and $b$ defined as $H(a, b)=\frac{2 a b}{a+b}$. In physically tangible terms, the selection criterion requires both links to have good channel conditions, which are not disparate. The Max-Min Selection Criterion (MM-RS) [9] also seeks the highest-gain link; it selects the specific link that combines the best gains between the SR and RD links, while excluding RNs that have bad channel conditions in one or both branches, which is formulated as:

$$
r=\arg \max _{i \in \mathcal{K}} \min \left(\left|h_{s, i}\right|^{2},\left|h_{i, d}\right|^{2}\right) .
$$

\section{EFFicient Relay SELECtion Methods}

Below a pair of new energy-efficient and lifetime-efficient (EE-LT) relay selection criteria are discussed.

\section{A. RS Method Based on Fuzzy Logic}

The Fuzzy Logic (FL) concept can be applied to problems that require non-binary decisions and solutions, while requiring a certain grade of flexibility in the treatment of variables; this goal may be achieved by combining conflicting design objectives, as detailed below. In the EE-LT relay selection context, fuzzy logic calculations may be carried out by appropriately combining values of both the normalized channel power gains $\left|h_{n}\right|^{2}$ of (3), and of the residual percentage power of each sensor $\left(E_{i}^{\%}\right)$ [10]. For each $\mathrm{RN}$, a numerical value given by the OF $f\left(\left|h_{n, i}\right|^{2}, E_{i}^{\%}\right)$ should be calculated and the $\mathrm{RN}$ having the highest $\mathrm{OF}$ is activated.

The Fuzzification Process starts by mapping the two variables into fuzzy sets by means of the qualitative evaluation, such as "low-full", "weak-strong" and so forth [11]. Thus, the variables $X_{1}$ and $X_{2}$ can be defined as the instantaneous channel gain and the remaining energy of the RN, respectively. The legitimate states are: $X_{1}=$ \{weak, average, strong\}; and $X_{2}=$ \{low, medium, full $\}$. Next, these variable states are mapped according to $f\left(\left|h_{n, i}\right|^{2}, E_{i}^{\%}\right)$ into the set: $Y=\{$ poor, inadequate, adequate, good, excellent $\}$. The mapping occurs through assignments of the type if $X_{1}$ assumes the state $a$ and $X_{2}$ assumes the state $b$ then $Y$ goes to state c. All possible implications can be summarized as:

\begin{tabular}{c|ccc}
\hline Variable & \multicolumn{3}{|c}{ Variable $E_{i}^{\%}$} \\
$\left|h_{n, i}\right|^{2}$ & Low & Medium & Full \\
\hline Weak & poor & inadequate & inadequate \\
Average & inadequate & adequate & good \\
Strong & adequate & good & excellent \\
\hline
\end{tabular}

In order to describe these linguistic elements, a generic trapezoidal function has been adopted [11]:

$$
\operatorname{trap}\left(x ; a, m_{1}, m_{2}, b\right)= \begin{cases}\frac{x-a}{m_{1}-a}, & \text { if } x \in\left[a, m_{1}\right] \\ 1, & \text { if } x \in\left[m_{1}, m_{2}\right] \\ \frac{b-x}{b-m_{2}}, & \text { if } x \in\left[m_{2}, b\right] \\ 0, & \text { otherwise }\end{cases}
$$

where $a$ and $b$ form the longer base (the bottom); $m_{1}$ and $m_{2}$ form the smaller base (the top), while ensuring that 
$a<m_{1} \leq m_{2}<b$. This trapezoidal function maps $x$ into the unit interval $[0 ; 1]$, indicating the degree of membership of $x$, i.e., $\mu(x)$. Thus, we can define the qualitative values for the variable $X_{1}$ as: "weak" for $h_{n} \leq m-\frac{m}{2}$ and "strong" for $h_{n} \geq m+\frac{m}{2}$. For $m-\frac{m}{2}<h_{n}<m+\frac{m}{2}$ a combination of the classifications "weak", "average" and "strong" is accomplished by considering its pertinence. Therefore, using trapezoidal functions for $X_{1}$ :

$$
\begin{aligned}
\text { weak } & =\operatorname{trap}\left(x_{1} ; 0,0, m-m / 2, m\right) ; \\
\text { average } & =\operatorname{trap}\left(x_{1} ; m-m / 2, m, m, m+m / 2\right) ; \\
\text { strong } & =\operatorname{trap}\left(x_{1} ; m, m+m / 2, \infty, \infty\right) ;
\end{aligned}
$$

In the same way, the variable $X_{2}$ may be classified as a function of the percentage of power remaining in the $\mathrm{RN}$, with the midpoint designated by $50 \%$ of the available charge:

$$
\begin{aligned}
\text { low } & =\operatorname{trap}\left(x_{2} ; 0,0,25,50\right) ; \\
\text { medium } & =\operatorname{trap}\left(x_{2} ; 25,50,50,75\right) ; \\
\text { full } & =\operatorname{trap}\left(x_{2} ; 50,75,100,100\right) ;
\end{aligned}
$$

In accordance with the ratings of $X_{1}$ and $X_{2}$, the degree of membership of $f\left(\left|h_{n, i}\right|^{2}, E_{i}^{\%}\right)$ in the range $\mu(x) \in[0 ; 100]$ is obtained as follows:

$$
\begin{aligned}
\text { poor } & =\operatorname{trap}(y ; 0,0,0,30) ; \\
\text { inadequate } & =\operatorname{trap}(y ; 10,30,30,50) ; \\
\text { adequate } & =\operatorname{trap}(y ; 30,50,50,70) ; \\
\text { good } & =\operatorname{trap}(y ; 50,70,70,90) ; \\
\text { excellent } & =\operatorname{trap}(y ; 70,100,100,100) ;
\end{aligned}
$$

Defuzzification Process. As a simple example, let us consider the case where a RN has $\left|h_{n, i}\right|^{2}=60$ and $E_{i}^{\%}=85$. Since its normalized channel gain is between $m$ and $m+\frac{m}{2}$, the classification of $X_{1}$ is given by the combination of strong and average, while the degree of membership associated with each of these sets is given by (15): $X_{1}=$ $\{($ average, 0.73$)$, (strong, 0.27$)\}$. Since this RN has an energy above the threshold amount for full, $X_{2}$ should be classified as $X_{2}=\{f u l l, 1\}$. In this example, where $X_{1}$ has two classifications, $Y$ is represented by the pair generated as the combination of $X_{1}$ and $X_{2}$; its membership degree is given by the lowest degree achieved by the function $\mu(y)$, i.e, $Y=\{(\operatorname{good}, \min (0.73,1)),($ excellent, $\min (0.27,1))\}=$ $\{($ good, 0.73$),($ excellent, 0.27$)\}$. Finally, the defuzzification step is performed through the center of gravity $(\mathrm{CoG})$ method [12] in the following steps:

a) Find the abscissa of the CoG of the geometric shape of each output fuzzy set. Since the functions are trapezoidal or triangular for the specific case of $m_{1}=m_{2}$, the abscissa of $\mathrm{CoG}$ can be readily calculated by: $C=$ $\frac{b^{2}+m_{2} \times b+m_{2}^{2}-m_{1}^{2}-a \times m_{1}-a^{2}}{3\left(b+m_{2}\right)-m_{1}-a}$.

b) For each output $Y_{i}$, calculate the area $A$ related to the degree of membership $\mu\left(Y_{i}\right)$ and the abscissa $f\left(\left|h_{n, i}\right|^{2}, E_{i}^{\%}\right)$.

c) Finally, the $\mathrm{CoG}$ is given by the weighted average of $\mathrm{CoG}=\sum_{j=1}^{\alpha} C_{j} \times A_{j} / \sum_{j=1}^{\alpha} A_{j}$, where $\alpha$ is the maximal number of combinations between $X_{1}$ and $X_{2}$.

For this example, the value of $\mathrm{CoG}=74.67$ is obtained.

\section{B. Minimum Energy Criterion (MEC) for Relay Selection}

DF Cooperative Mode. The energy-per-bit in a cooperative DF relay mode between the source and sink relayed by the $i$-th RN can be obtained by substituting (10) and (11) into (6), resulting in [4] $E_{i}^{\mathrm{coo}}=$

$$
\begin{aligned}
& =\frac{f(2 \mathcal{S})}{2 \mathcal{S B}}\left(\frac{1}{\left|h_{s, i}\right|^{2}}+\frac{\zeta}{\left|h_{d, i}\right|^{2}}-\frac{\left|h_{s d}\right|^{2}}{\left|h_{s, i}\right|^{2}\left|h_{i, d}\right|^{2}}\right)+\frac{(1+\zeta) P_{C}}{2 \mathcal{S B}} \\
& \text { where } f(\mathcal{S})=\frac{2 \varrho N_{0} B}{3 \mathcal{G}}\left(2^{\mathcal{S} / \varrho}-1\right) \ln \left(\frac{1+\sqrt{1-P_{e}}}{5 P_{e}}\right)
\end{aligned}
$$

The transmitted node powers required for meeting the QoS constraint in terms of the maximal BER in the UL and DL are expressed by:

$$
\begin{array}{ll}
\text { UL-S: } & P_{\mathrm{S}}^{\mathrm{U}}=f(2 \mathcal{S})\left|h_{s, i}\right|^{-2} \\
\text { UL-R: } & P_{\mathrm{R}}^{\mathrm{U}}=f(2 \mathcal{S})\left|h_{i, d}\right|^{-2}\left(1-\left|h_{s, d}\right|^{2}\left|h_{s, i}\right|^{-2}\right) \\
\text { DL-R: } & P_{\mathrm{R}}^{\mathrm{D}}=f(2 \mathcal{S})\left|h_{s, i}\right|^{-2}\left(1-\left|h_{s, d}\right|^{2}\left|h_{i, d}\right|^{-2}\right) .
\end{array}
$$

Direct Mode. The transmissions may take place directly, without the use of a relay. In this case, the minimum required energy-per-bit is:

$$
E^{\mathrm{dir}}=-\frac{2 \zeta \varrho N_{0} \ln \left(5 P_{e}\right)}{3\left|h_{s, d}\right|^{2} \mathcal{G S}}\left(2^{\mathcal{S} / \varrho}-1\right)+\frac{\zeta P_{C}}{\mathcal{S} B} .
$$

Minimum Energy Criterion. The total energy-per-bit in (18) can be optimized by choosing the best RN $\left(\iota^{*}\right)$ that minimizes $E_{i}^{\text {coo }}$. This criterion may be formulated as:

$$
\begin{aligned}
& \iota^{*}=\arg \min _{i \in\{1,2, \ldots, K\}}\left(\frac{1}{\left|h_{s, i}\right|^{2}}+\frac{\zeta}{\left|h_{d, i}\right|^{2}}-\frac{\left|h_{s d}\right|^{2}}{\left|h_{s, i}\right|^{2}\left|h_{i, d}\right|^{2}}\right) \\
& \text { s.t. }\left(\text { (c.1) }: f(2 \mathcal{S})\left|h_{s, i}\right|^{-2}+P_{C} \leq P_{\max } ;\right. \\
& \quad \text { (c.2) }: \frac{f(2 \mathcal{S})\left(\left|h_{s, i}\right|^{2}-\left|h_{s, d}\right|^{2}\right)}{\left|h_{s, i}\right|^{2} \cdot\left|h_{i, d}\right|^{2}}+P_{C} \leq P_{\max } ;
\end{aligned}
$$

Note that the condition $\left(P_{\max }-P_{C}\right) \cdot\left|h_{s, i}\right|^{2} \geq f(2 \mathcal{S})=\mathrm{p}_{\mathrm{s}}^{\text {th }}$ formulated in the first constraint of (21) has to be met for the $i$ th relay-candidate to guarantee the QoS BER requirement in the source-relay hop; $\mathrm{p}_{\mathrm{S}}^{\text {th }}$ is the minimum receiver power required for achieving the QoS BER requirement, when using DF cooperation combined with a coding scheme. Analogously, constraint (C.2) quantifies the channel gain required for the $i$ th relay to meet the QoS BER requirement:

$\left(P_{\max }-P_{C}\right) \cdot\left|h_{i, d}\right|^{2} \geq f(2 \mathcal{S})\left(1-\left|h_{s, d}\right|^{2} /\left|h_{s, i}\right|^{2}\right)=\mathrm{p}_{\mathrm{R}}^{\text {th }}$.

Finally, the transmitted power of the SN and of the selected $\mathrm{RN}$ can be immediately determined from the channel gain and from the corresponding minimum received power required for achieving our QoS BER requirement under DF cooperation combined with a coding scheme; from (19a) and (19b) we readily obtain:

$$
P_{\mathrm{S}}^{\mathrm{U}}=\mathrm{p}_{\mathrm{S}}^{\mathrm{th}}\left|h_{s, i}\right|^{-2} ; \quad \text { and } \quad P_{\mathrm{R}, i}^{\mathrm{U}}=\mathrm{p}_{\mathrm{R}}^{\mathrm{th}}\left|h_{i, d}\right|^{-2} .
$$

Cooperative Region Determination using MEC Optimization. Based on the concept of the cooperative regions discussed in Section I-B, the beneficial cooperative region and the best RN location can be analytically determined following the MEC [4]. Hence, based on (12) we can determine the energy saving $E_{\text {save }}=\frac{E^{\text {dir }}-E^{\text {coo }}}{E^{\text {dir }}}$, with $E^{\text {dir }}$ and $E^{\text {coo }}$ obtained accordingly from (20) and (18). 


\section{NUMERICAL RESULTS}

Simulation Scenario: a rectangular $500 \times 600\left[\mathrm{~m}^{2}\right]$ cell relying on $K$ relays randomly and uniformly distributed on the ellipsoid region defined by the cooperative energy-gain region of $E_{\text {save }}>0$ is considered. The SN and sink nodes are 600 meters away from each other and their communication was supported by activating a single relay in a two-hop communication scenario. Trellis coded modulation has been employed, hence the transmission power necessary for guaranteeing a sufficiently low $P_{\mathrm{e}}$ was given in (10), where both the code-rate of the encoder and the trellis coding gain have been taken into account, when calculating the necessary transmit power level. Table I shows the main parameters adopted in our numerical simulations.

TABLE I

SIMULATION PARAMETERS

\begin{tabular}{ll}
\hline Parameter & Value \\
\hline Protocol & Coding DF \\
Encoder & Trellis \\
Number of Relays & $K \in[2 ; 30]$ \\
Coop. Spectral efficiency & $\mathcal{S}=1[\mathrm{bps} / \mathrm{Hz}]$ \\
Traffic Asymmetry Factor & $\zeta \in[0.10 ; 1.0]$ \\
Channel constant & $\kappa=-128.1[\mathrm{~dB}]$ \\
System Bandwidth & $B=180 \mathrm{KHz}$ \\
Carrier frequency & $f_{c}=1.8 \mathrm{GHz}$ \\
Noise PSD & $N_{0}=-171[\mathrm{dBm} / \mathrm{Hz}]$ \\
Path loss exponent & $\xi=3.76$ \\
Coding gain & $\mathcal{G}=4.7[\mathrm{~dB}]$ \\
Coding rate & $\varrho=2 / 3$ \\
Maximum BER & $P_{e}=10^{-4}$ \\
Max. Battery Power & $P_{\max }=33[\mathrm{dBm}]$ \\
Initial energy of each relay & $\varepsilon_{0}=10[\mathrm{~J}]$ \\
Circuitry power & $P_{C}=20[\mathrm{dBm}]$ \\
S-D distance & $d_{s, d}=0.6[\mathrm{~km}]$ \\
\hline
\end{tabular}

The RS algorithms of Section I-C were characterized in terms of the amount of energy-per-bit $E_{\mathrm{B}}$ required for maintaining the target BER and the network lifetime $\mathcal{L}$. All RNs had the same amount of initial energy $\varepsilon_{0}$, and at each iteration $i$ we assumed a new location for the RNs associated with a channel gain according to its new position. The RN selected by the respective algorithm transmits a frame constituted by a total of 512 bits; Then, the selection criterion is re-applied. Each transmission dissipates one lifetime unit [ltu], i.e., $\mathcal{L}=$ $1[\mathrm{ltu}] \equiv 512$ [bits]. The time required for transmitting this frame is simply $\tau_{\mathcal{L}}=\frac{\mathcal{L}}{R}$ [sec]. This process is continued until the first $\mathrm{RN}$ has $\varepsilon_{j} \leq 0$, i.e. it exhausts its energy resource.

Furthermore, we assume that the information successfully decoded by the selected RN with no errors is also successfully decoded at the DN. Hence, for simplifying our analysis, no retransmission ${ }^{1}$ of the information is assumed. Thus, the overall amount of received information is assumed to be identical to the transmitted amount given by $\mathcal{I}=512 \cdot \mathcal{L}$ [bits].

A total of 20,000 iterations were performed, and the average values of both figures of merit $\left(E_{\mathrm{B}}\right.$ and $\left.\mathcal{L}\right)$ are displayed in Fig. 3. The traffic asymmetry factor values of $\zeta=0.1, \zeta=0.5$ e $\zeta=0.95$ were adopted.

\footnotetext{
${ }^{1}$ There is no need for retransmission, since the DF protocol and trellis coding are used with the goal of maintaining the minimum transmission power required for guaranteeing a maximum bit-error probability, $P_{e}$.
}

The MHM-RS and MaxMin relay selection (MM-RS) algorithms of Section I-C impose a low energy per bit dissipation, since both focus on RS algorithms purely based on the channel gain. Consequently, the RNs experiencing better conditions tend to be repeatedly selected, hence reducing the network's lifetime.
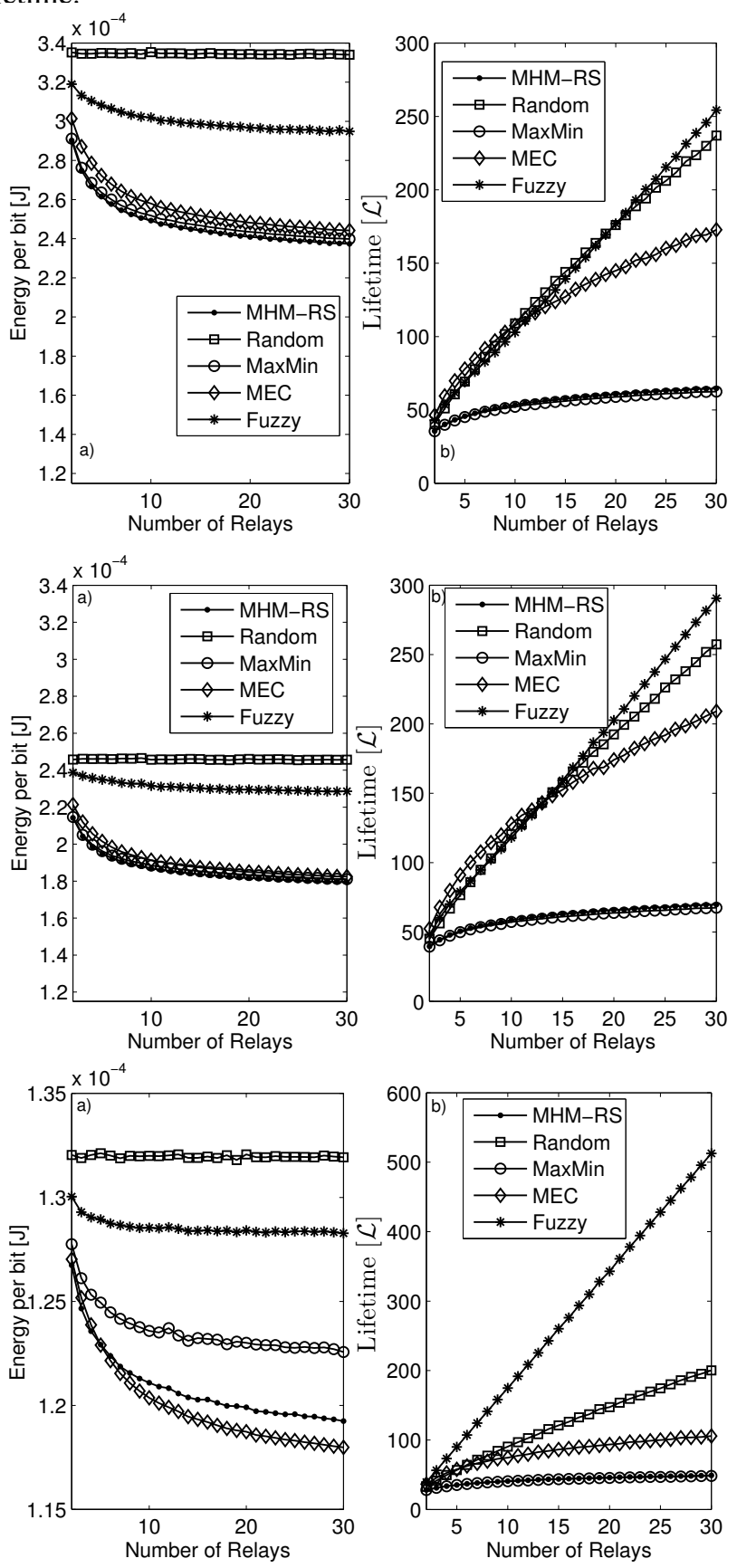

Fig. 3. a) Energy-per-bit consumption, and b) Lifetime v.s. the number of relays. Traffic factor $\zeta=0.95 ; \zeta=0.50 ; \zeta=0.10$, respectively.

When randomly selecting relays, with no further selection criteria imposed, the R-RS algorithm distributes the energy equally; as a result, this increases the power-per-bit dissipation, since the RNs experiencing hostile channel conditions are likely to be chosen, which increases the system's overall energy consumption. However, by distributing the task of relaying equally to all relays, the probability that a $\mathrm{RN}$ prematurely exhausts its energy reserve becomes low; as a consequence, this algorithm achieves a high lifetime. 
The MEC algorithm of Section II-B selects the specific RN, where the relative energy-per-bit is the lowest possible. However, the constraints in (21) prevent any RN from depleting its energy reserves prematurely. By checking how much the next bit-frame's transmission would deplete the energy reserve of a specific relay-candidate, the MEC algorithm excludes this $\mathrm{RN}$ from the selection process; in doing so, another relaycandidate potentially experiencing a lower channel gain and thus imposing a higher energy consumption but having a high energy reserve is likely to be selected. Thus, the energy consumption is increased slightly in comparison to the MHMRS and MM-MS algorithms. However, the network's lifetime improves considerably under the MEC RS criterion.

The fuzzy logic-based RS (FL-RS) algorithm aims for balancing the optimization of the $E_{\mathrm{B}}$ and $\mathcal{L}$ criteria. We can see from from Fig. 3 that the FL-RS algorithm fails to achieve a reduced energy-per-bit in the interest of prolonging the network's lifetime: for highly asymmetric DL-oriented traffic $(\zeta=0.1)$, the FL-RS algorithm's lifetime is substantially higher than that of all other criteria considered. When the traffic is symmetric $(\zeta=0.5)$, or UL-oriented $(\zeta=0.95)$, both the FL-RS and R-RS algorithm's lifetime is similar, as long as the number of relays is in the range of $K \in[1 ; 20]$. Finally, for $K>20$ relays operating in symmetric traffic, the FL-RS algorithm also exhibits a superior lifetime.

The steps necessary for implementing the FL-RS can be performed at a complexity order of $\mathcal{O}(K)$, except for the ordering of the resultant vector-elements, which has a complexity order of at least $\mathcal{O}(K \log K)$ [13]. For the remaining algorithms the process of ordering vectors for obtaining maximum or minimum values tends to impose the highest complexity.

As we can see from Fig. 3 when varying the traffic asymmetry factor $\zeta$, all the RS algorithms exhibit a similar behavior regarding the network lifetime. On the other hand, different tendencies prevail regarding the energy-per-bit, when the traffic becomes more asymmetric in the DL-oriented traffic direction. In order to demonstrate this behavior, both the energy per bit required and the network lifetime attained were analyzed as a function of $\zeta$ for a fixed number of $K=10 \mathrm{RNs}$ in Fig. 4. Note that for $\zeta \leq 0.4$, the energy-per-bit required by the MEC algorithm is lower than that of the MHM-RS and RS-MM algorithms. However, the corresponding lifetime is reduced, when the traffic-asymmetry obeys $\zeta \rightarrow 0$. It is worth noting that the FL-RS criterion achieves the highest steady lifetime for all traffic-asymmetry configurations.

\section{CONCLUSIONS}

We examined the impact of geographic relay node positions and RS algorithms both on the energy efficiency and on the overall wireless sensor network lifetime. Several relay selection criteria have been applied, while aiming for the same spectral efficiency as direct mode communication. The numerical results generated for different traffic asymmetry factors $\zeta$ confirm the low energy-per-bit requirement of both the classic MHM-RS and MM-RS algorithms, which is achieved at the cost of its fragility in terms of reduced network lifetime.

The recently proposed MEC criterion is capable of remarkably improving the network's lifetime, while the associated energy-per-bit remains acceptable, making this criterion more balanced in terms of its LT and EE. The FL-based RS algorithm has been demonstrated to be the best choice, since it is capable of maximizing the lifetime, while maintaining the energy-per-bit required at reasonable levels. Its performance is considerably higher than that of the R-RS algorithm in terms of its energy-per-bit required, while demonstrating a superior lifetime performance for diverse number of relays and traffic asymmetry factors.
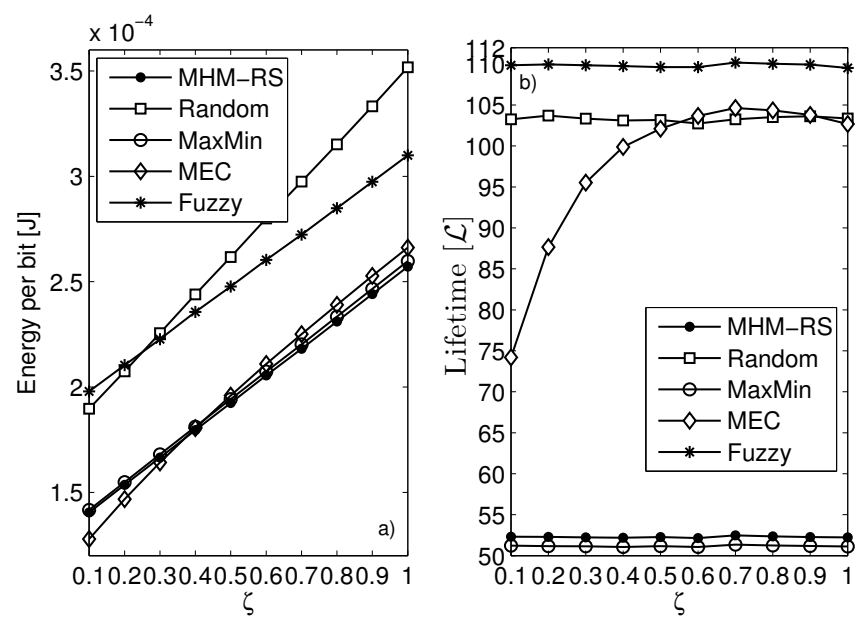

Fig. 4. WSN performance under different RS criteria; $K=10$ relays and different asymmetric traffics $\zeta$ : a) Energy consumption per bit; b) Lifetime.

\section{REFERENCES}

[1] Y. Chen, W. Shen, H. Huo, and Y. Xu, "A smart gateway for health care system using wireless sensor network," in Fourth International Conference on Sensor Technologies and Applications, pp. $545-550$, july 2010 .

[2] I. Akyildiz, W. Su, Y. Sankarasubramaniam, and E. Cayirci, "Wireless sensor networks: a survey," in Computer Networks, vol. 38, pp. 393-422, Elsevier, 2002.

[3] A. Goldsmith, Wireless Communications. Cambridge University Press, 2005.

[4] W. Yang, L. hua Li, W. lu Sun, and Y. Wang, "Energy-efficient relay selection and optimal relay location in cooperative cellular networks with asymmetric traffic," The Journal of China Universities of Posts and Telecommunications, vol. 17, no. 6, pp. 80 - 88, 2010.

[5] T. Hunter and A. Nosratinia, "Diversity through coded cooperation," IEEE Trans. Wireless Communications, vol. 5, pp. 283-289, Feb. 2006.

[6] L. Vandendorpe, R. Duran, J. Louveaux, and A. Zaidi, "Power allocation for ofdm transmission with df relaying," in Communications, 2008. ICC '08. IEEE International Conference on, pp. 3795 -3800, may 2008.

[7] K. Zarifi, M. Abuthinien, A. Ghrayeb, and S. Affes, "Relay selection schemes for uniformly distributed wireless sensor networks," in WCNC'09 - IEEE Wirel. Commun. \& Netw. Confer., pp. 1-6, Apr. 2009.

[8] X. Chen, T. Siu, Q. Zhou, and F. Lau, "High-SNR analysis of opportunistic relaying based on the maximum harmonic mean selection criterion," IEEE Signal Process. Lett., vol. 17, pp. 719 -722, aug. 2010.

[9] I. Krikidis, "Relay selection for two-way relay channels with MABC DF: A diversity perspective," IEEE Transactions on Vehicular Technology, vol. 59, pp. $4620-4628$, nov. 2010.

[10] G. de Santi Peron, G. G. de Oliveira Brante, and R. D. Souza, "Distributed method of relays selection in cooperative networks using fuzzy logic to optimization flow and lifetime (In Portuguese), ' in SBrT'11 XIX Simpósio Brasileiro de Telecomunicações, pp. 1-5, Oct. 2011.

[11] M. Ganesh, Introduction To Fuzzy Sets And Fuzzy Logic. Prentice-Hall Of India Pvt. Limited, 2006.

[12] D. Huang, J. Yang, X.-M. Li, and C.-L. Li, "An algorithm for generating fuzzy decision tree based on center of gravity," in Machine Learning and Cybernetics, 2008 International Conference on, vol. 1, pp. $485-489$, july 2008.

[13] Y. Yang, P. Yu, and Y. Gan, "Experimental study on the five sort algorithms," in Mechanic Automation and Control Engineering (MACE), 2011 Second International Conference on, pp. 1314 -1317, july 2011. 